\title{
Costo efectividad de la vacunación contra influenza en menores de 2 años y mayores de 65 años en Colombia
}

\section{Cost effectiveness of influenza vaccination in children under 2 years old and elderly in Colombia}

Alexandra Porras-Ramírez ${ }^{1}$, Nelson Alvis-Guzmán ${ }^{2}$, Alejandro Rico-Mendoza ${ }^{1}$, Luís Alvis-Estrada ${ }^{3}$, Carlos A. Castañeda-Orjuela ${ }^{1}$, Martha P. Velandia-González ${ }^{4}$ y

Fernando de la Hoz-Restrepo ${ }^{1}$

1Facultad de Odontología.Universidad deAntioquia.Medellín-Colombia. agudeloandres@odontologia.udea.edu.co

2 Área de Medicina Preventiva y Salud Pública. Universidad de Alicante. Alicante. España.

diana.gil@ua.es, carmen.vives@ua.es, elena.ronda@ua.es

Recibido 26 Febrero 2009/Enviado para Modificación 15 Julio 2009/Aceptado 20 Agosto 2009

\section{RESUMEN}

Objetivo Estimar la carga de enfermedad asociada a influenza y modelar el impacto epidemiológico y económico de la introducción de la vacuna para influenza en Colombia. Métodos Se realizó un estudio de evaluación económica completa de la introducción de la vacuna de influenza en dos grupos poblacionales. Los desenlaces seleccionados en menores de dos años fueron la frecuencia de enfermedad respiratoria (ERA), consultas y hospitalizaciones por ERA. En mayores de 65 años se adicionaron las muertes y hospitalizaciones por enfermedad cardiosvascular y cerebrovascular.

Resultados En el escenario sin vacunación, el virus de influenza produciría anualmente 4 300 casos, 2700 consultas, 900 hospitalizaciones y 230 muertes por ERA en menores de dos años. En mayores de 65 años, se presentarían anualmente 670 muertes por neumonía, 1150 muertes por enfermedad cardiovascular y 720 muertes por enfermedad cerebrovascular relacionadas con influenza. El costo efectividad de la vacuna en menores de dos años oscila entre US \$1900 y US \$ 2967 por muerte evitada mientras que para mayores de 65 años la razón de costo efectividad seria costo ahorrativa.

Conclusiones Los resultados del presente estudio apoyarían la decisión del Ministerio de la Protección Social y algunas Secretarias de Salud de introducir la vacunación en Colombia para menores de dos años y mayores de 65 años.

Palabras Clave: Influenza, vacunación, costo efectividad, Colombia (fuente: DeCS, BIREME).

\section{ABSTRACT}

Objective Estimating the burden of disease associated with influenza virus season and modelling the epidemiological and economic impacts of introducing an inactivated vaccine to Colombia. 
Methodology A complete economic evaluation was done in children aged less than 2 and adults aged over 65. The outcomes evaluated in the under 2-year olds included: the yearly number of cases of acute respiratory infection (ARI), medical visits, hospitalisations and deaths by ARI. The outcomes measured in adults were the number of yearly deaths and hospitalisations due to cardiocirculatory diseases (CCD).

Results Influenza infection in children under 2 years old not having had vaccination may cause 4,300 cases, 2,700 medical visits, 900 hospitalisations and 230 deaths by ARI yearly. Amongst the elder group, influenza infection would be associated with 670 deaths by pneumonia and 1,870 deaths from CCD. The incremental cost effectiveness ratio (ICER) for flu vaccination among children under 2 ranged from USD \$1,900 to USD\$ 2,967 per averted death. ICER was cost saving in adults aged over 65 .

Conclusions This study's results supported the Colombian Ministry of Health's initiative for introducing yearly flu vaccination amongst small children and older adults in Colombia.

Key Words: Influenza, vaccination, cost effectiveness, Colombia (source: MeSH, NLM).

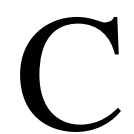

omo problema de salud pública, la influenza genera, en los países industrializados, una carga económica considerable en cuanto a costos de atención en salud (1), pérdida de días laborales y/o escolares. En una reciente revisión sobre ausentismo laboral en pacientes con influenza el promedio de pérdida de días fue de 5.9 \pm 4.7 (2). Igualmente cálculos recientes en Estados Unidos con base en la población 2003, indican que anualmente la epidemia de influenza genera en promedio 610660 años de vida perdidos (YLL), 3,1 millones de días de hospitalización y 31,4 millones de consultas médicas. Los costos directos de atención en promedio son de U\$10,4 billones (IC95 \% U \$4,1U $\$ 22,2$ ) anualmente y la carga económica total anual de la influenza epidémica se estima en U\$87, 1 billones (IC95 \%: U\$47,2-U\$149,5) (1).

La influenza tiene una distribución mundial, con una tasa de ataque anual estimada entre $5 \%$ y $10 \%$ en adultos y entre $20 \%$ y $30 \%$ en niños. Los brotes epidémicos ocurren principalmente durante la estación invernal en climas templados y de forma menos predecible en las regiones tropicales (3). En Colombia, la primera epidemia registrada y documentada virológicamente de influenza se presentó en Ambalema (Tolima) en abril de 1994 cuando se pasó de forma repentina de un promedio normal de 7 a 10 consultas semanales por problemas respiratorios a 200 consultas en una semana. De 6278 personas censadas, la tasa de ataque fue del $40 \%$ debido al virus influenza humana A/Beijing/32/92 (H3N2) (4).

Las tasas más altas de infección se observan en niños de 5 a 9 años de edad, pero la morbilidad más grave y la mortalidad por influenza ocurren con 
mayor frecuencia en los menores de 2 años de edad, los mayores de 65, y las personas con factores de alto riesgo como la enfermedad pulmonar o cardiovascular y diversos tipos de inmunodepresión (5). La medida más eficaz para disminuir el impacto de la infección estacional con influenza es la vacunación, la cual se realiza con una vacuna inactivada que contiene los virus que mas probablemente circularan en cada estación. Sin embargo, la composición vira de la vacuna debe ser cambiada frecuentemente y los grupos de riesgo deben ser vacunados anualmente ya que los anticuerpos despertados por la vacuna bajan después de 9 a 11 meses. Esta vacuna se usa ampliamente en países del hemisferio norte y sur pero mucho menos en los países intertropicales debido a la escasez de datos epidemiológicos y del impacto del virus y de la efectividad de la vacunación. (6-8).

En el presente estudio se evalúa el impacto epidemiológico y económico de la introducción de la vacuna contra influenza estacional en menores de 2 años y adultos mayores de 65 años en Colombia.

\section{MÉTODOS}

Se combinaron dos tipos de estudios. Primero, se estimo la carga de enfermedad tanto en niños como en adultos y, seguidamente se estimó el costo efectividad de la introducción de la vacuna en ambas poblaciones.

Carga de enfermedad por influenza en menores de 2 años

Para los menores de 2 años se tuvo en cuenta como denominador poblacional por departamento el estimado por el Programa Ampliado de Inmunizaciones (PAI) ajustado según la tasa de mortalidad infantil en hombres. Los datos de incidencia de enfermedad respiratoria debida a influenza en menores de 2 años se tomaron de la literatura y la tasa de mortalidad atribuible a Influenza fue estimada por los investigadores (9). La tasa de incidencia según Borrero y colaboradores (10), para IRA baja por Influenza fue de 0,56 x 1000 niños mes, para IRA alta de 1,68 x 1000 niños mes y para ERA total de 2,24 x 1000 niños mes. A partir de ello se calcularon los casos por Influenza los cuales se ajustaron según la ERA reportada en la Encuesta Nacional de Demografía y Salud 2005 -ENDS(11).

Para calcular las hospitalizaciones esperadas por neumonía atribuible a Influenza se tomaron las tasas estimadas por Chiu et al (4), 0,74 x 1000 personas año (IC $95 \%$ 0,35-1,29). Este número se ajustó por accesibilidad, 
usando las consultas reportadas en la ENDS por departamento. El número de muertes esperadas se calculó utilizando la tasa de mortalidad hallada por Porras et al (9). Se utilizaron dos estimadores: la tasa de mortalidad de Bogotá $(0,21$ x 100000 niños años, IC $95 \% 0.19-0.39)$ y la tasa de mortalidad de Manizales (0,13 x 100.000 niños año, IC $95 \%$ 0.10-0.43). El número de muertes esperadas por departamento se ajustó teniendo en cuenta la razón de mortalidad infantil de cada Departamento y su relación con la mortalidad infantil de Bogotá.

Carga de enfermedad por influenza en mayores de 65 años

Para los adultos mayores de 65 años, se tuvo en cuenta el denominador poblacional proyectado por el DANE para el año 2007. La estimación del número de muertes se hizo a partir del estudio de Porras et al (9) donde se calcularon tasas de mortalidad por neumonías, enfermedad cardiovascular y cerebrovascular para adultos mayores en Bogotá y Manizales. Las tasas de mortalidad por neumonías en las dos ciudades fueron de $0,30 \times 10^{5}$ (IC $95 \% 0,21-0,45$ ) en Bogota y de $0,47 \times 10^{5}$ (IC $95 \% 0,33-0,54$ ) en Manizales. Para enfermedad cardiovascular la tasa de mortalidad en Bogotá fue de 0,53 x $10^{5}$ (IC $95 \% 0,48-0,65$ ) y la de Manizales fue $0,51 \times 10^{5}$ (IC $95 \% 0,47-0,76$ ). Para enfermedad cerebrovascular la tasa de Bogotá fue de $0,32 \times 10^{5}$ (IC $95 \% 0,30-0,45$ ) y para Manizales fue de $0,62 \times 10^{5}$ (IC $95 \% 0,54-0,70$ ).

Análisis de costo efectividad

Usando un modelo de árbol de decisiones se simularon las situaciones con y sin programa de vacunación para ambos grupos poblacionales. La eficacia de la vacuna se tomo de los resultados de un meta análisis desarrollado por Moreno et al (12), en el que la protección contra hospitalización durante periodos de influenza fluctuaba entre $20 \%$ y $24 \%$ en estudios de cohorte y entre $41 \%$ y $43 \%$ para estudios de casos y controles. Además, la protección contra muerte durante un periodo de influenza iba entre $42 \%$ y $58 \%$.

Desde la perspectiva del tercer pagador, se estimaron los costos de la carga de enfermedad usando los parámetros de costo medio. La fuente de datos de costos fueron sendos estudios de microcosteo de pacientes hospitalizados menores de dos años con diagnóstico de neumonía. Los resultados de estancias y costos fueron similares a los estimados para países de ingreso medio (6-10). Los costos de vacunación incluyen los costos de adquisición y aplicación de la vacuna. Los costos para vacuna aplicada en 
menores de dos años es de 4 a 6 dólares y para mayores de 65 años son de 7 a 9 dólares.

El modelo estableció los Años de Vida Ajustados por Discapacidad (AVAD) evitados, las muertes evitadas, las hospitalizaciones y las atenciones ambulatorias evitadas. Además se estimaron los ratios de costo efectividad incremental (ICER, por su sigla en inglés), los cuales están dados por la valoración de lo que representa agregar costos adicionales de vacunación para intentar salvar años de vida de relacionados con la carga de enfermedad por rotavirus. Tanto los costos como los resultados se expresan en dólares americanos de 2007 y fueron descontados a una tasa de $3 \%$.

Fuente de financiación y su papel en el estudio: El estudio fue financiado en su totalidad por el Ministerio de la Protección Social. A través del Programa Ampliado de Inmunizaciones (PAI) el Ministerio participo en la interpretación de los resultados del modelo y en la redacción de este articulo

\section{RESULTADOS}

Carga de enfermedad por Influenza en menores de 2 años

En la cohorte no vacunada se presentarían 4310 casos de enfermedad respiratoria aguda por influenza (IC $95 \% 2559-14$ 181) mientras que las consultas medicas por esta causa, ajustadas por accesibilidad, serían 2717 casos (IC $95 \% 1613-8$ 941). Las hospitalizaciones por ERI baja atribuibles a influenza serian 926 (IC95 \% 428-1 579) mientras que las muertes esperadas con la tasa de mortalidad deBogotá serian 384 (IC $95 \%$ 347-712) y con la tasa de Manizales 237 (IC $95 \%$ 183-785).

Carga de enfermedad por Influenza en adultos mayores de 65 años.

Las muertes por neumonía atribuible a Influenza serían 678 si se usa la tasa de mortalidad de Bogotá (IC $95 \%$ 475-1 017) mientras que si se usa la de Manizales se esperarían 1063 (IC $95 \%$ 746-1 221). Las muertes esperadas por enfermedad cardiovascular serian 1198 (IC95 \% 1 085-1 469) usando la tasa de Bogotá y 1 153 (IC95 \% 1 063-1 718), usando la tasa de Manizales.

Por enfermedad cerebrovascular se esperarían 723 muertes (IC $95 \%$ 678-1 017) usando la tasa de Bogotá y 1402 (IC95 \% 1 221-1 583), usando la tasa de Manizales. Las hospitalizaciones por las tres causas anteriores se 
calculan en 24743 anuales (IC95 \% 16805 - 34763 hospitalizaciones). Análisis económico de la introducción de la vacuna contra influenza

Los costos de la enfermedad asociada a infección por el virus de la influenza estarían entre 85,2 y 178,4 millones de dólares anuales (Tabla 1). Vacunar una cohorte de menores de un año con coberturas de $90 \%$ generaría costos por U\$ 4183335 y vacunar la población de mayores de 65 años con coberturas de $80 \%$ costaría U\$ 14688 589. La aplicación de la vacuna reduciría los costos de la carga anual de enfermedad asociada a influenza a un valor entre 35,7 y 74,2 millones de dólares por lo que se evitarían entre 49,5 y 104,1 millones de dólares, $59 \%$ de los costos de la carga.

En cuanto a los resultados de costo efectividad, en menores de dos años resulta ser muy costo efectiva considerando los parámetros de OMS (19) y en adultos mayores de 65 años la vacunación resulta ser costo ahorrativa. En el análisis de sensibilidad univariado que se realizo usando los escenarios de Bogota y Manizales la vacunación contra influenza siguió siendo costo efectiva tanto en menores de dos años como en adultos mayores de 65 años. (Tabla 2).

\section{DISCUSIÓN}

Este es el primer estudio realizado en la zona tropical de las Américas que muestra el impacto económico y epidemiológico de la circulación del virus de influenza para adultos mayores de 65 años y niños menores de dos años, las dos poblaciones más susceptibles y donde la enfermedad resulta siendo causante de un número importante de hospitalizaciones y muertes.

El presente trabajo tomó como estudio de referencia para los niños menores de 2 años el realizado por Borrero et al (10), en el que se realizó el seguimiento de una cohorte de niños menores de 17 meses de estrato socioeconómico bajo en la ciudad de Cali durante 2 años. La incidencia de influenza en esa cohorte sugiere que durante el periodo de seguimiento la actividad de influenza no fue muy alta, lo que puede llevar, en nuestro estudio, a una subestimación del impacto epidemiológico y económico del virus de influenza. Por ello, consideramos que los cálculos arrojados en nuestro estudio son estimados conservadores del verdadero impacto ocasionado por el virus de influenza en Colombia. 
Tabla 1. Costos por ERA asociada a Influenza con y sin vacunación. Colombia 2007 (en miles de dólares del 2007)

\begin{tabular}{|c|c|c|c|c|c|c|}
\hline Escenarios & Casos & $\begin{array}{c}\text { Lim } \\
\text { inf } \\
\end{array}$ & $\begin{array}{l}\text { Lim } \\
\text { sup }\end{array}$ & Costos & Lim inf & Lim sup \\
\hline \multicolumn{7}{|l|}{ Escenario sin vacunación } \\
\hline $\begin{array}{l}\text { Consultas por ERA } \\
\text { ajustadas en<de } 2 a\end{array}$ & 2717 & 1613 & 8941 & 299,8 & 177,9 & 986,6 \\
\hline $\begin{array}{l}\text { Hospitalizaciones por ERA } \\
\text { ajustadas en<de } 2 a\end{array}$ & 906 & 428 & 1579 & 946,5 & 321,9 & 2062,1 \\
\hline $\begin{array}{l}\text { Muertes por ERA según } \\
\text { Bogotá en<de } 2 a\end{array}$ & 435 & 394 & 809 & & & \\
\hline $\begin{array}{l}\text { Muertes por ERA según } \\
\text { Manizales en<de } 2 a\end{array}$ & 237 & 183 & 785 & & & \\
\hline $\begin{array}{l}\text { Hospitalizaciones por ERA } \\
\text { ajustadas en }>65 a\end{array}$ & $\begin{array}{l}24 \\
743\end{array}$ & $\begin{array}{c}16 \\
805\end{array}$ & $\begin{array}{c}34 \\
763\end{array}$ & 124835,8 & 84786,2 & 175389,7 \\
\hline $\begin{array}{l}\text { Muertes por ERA según } \\
\text { Bogotá en>de } 65 a\end{array}$ & 678 & 475 & 1017 & & & \\
\hline $\begin{array}{l}\text { Muertes por ERA según } \\
\text { Manizales en>de } 65 a\end{array}$ & 1063 & 746 & 1221 & & & \\
\hline Totales & & & & 126082,2 & 85286,2 & 178438,6 \\
\hline \multicolumn{7}{|l|}{ Escenario con vacunación } \\
\hline $\begin{array}{l}\text { Consultas por ERA } \\
\text { ajustadas en<de } 2 a\end{array}$ & 598 & 355 & 1967 & 65,9 & 39,1 & 217,0 \\
\hline $\begin{array}{l}\text { Hospitalizaciones por ERA } \\
\text { ajustadas en<de } 2 a\end{array}$ & 199 & 94 & 347 & 208,2 & 98,3 & 362,9 \\
\hline $\begin{array}{l}\text { Muertes por ERA según } \\
\text { Bogotá en<de } 2 a\end{array}$ & 339 & 307 & 631 & & & \\
\hline $\begin{array}{l}\text { Muertes por ERA según } \\
\text { Manizales en<de } 2 a\end{array}$ & 185 & 143 & 612 & & & \\
\hline $\begin{array}{l}\text { Hospitalizaciones por ERA } \\
\text { ajustadas en }>65 a\end{array}$ & $\begin{array}{c}10 \\
392\end{array}$ & 7058 & $\begin{array}{c}14 \\
600\end{array}$ & 52431,0 & 35610,2 & 73663,7 \\
\hline $\begin{array}{l}\text { Muertes por ERA según } \\
\text { Bogotá en>de } 65 a\end{array}$ & 393 & 276 & 590 & & & \\
\hline $\begin{array}{l}\text { Muertes por ERA según } \\
\text { Manizales en>de } 65 a\end{array}$ & 627 & 440 & 720 & & & \\
\hline Totales & & & & 52705,2 & 35747,7 & 74243,7 \\
\hline \multicolumn{7}{|l|}{ Costos y eventos evitados } \\
\hline $\begin{array}{l}\text { Consultas por ERA } \\
\text { ajustadas en }<\text { de } 2 a\end{array}$ & 2119 & 1258 & 6974 & 233,8 & 138,8 & 769,5 \\
\hline $\begin{array}{l}\text { Hospitalizaciones por ERA } \\
\text { ajustadas en<de } 2 a\end{array}$ & 707 & 334 & 1232 & 738,3 & 2236 & 1699,2 \\
\hline $\begin{array}{l}\text { Muertes por ERA según } \\
\text { Bogotá en<de } 2 a\end{array}$ & 96 & 87 & 178 & & & \\
\hline $\begin{array}{l}\text { Muertes por ERA según } \\
\text { Manizales en<de } 2 a\end{array}$ & 52 & 40 & 173 & & & \\
\hline $\begin{array}{l}\text { Hospitalizaciones por ERA } \\
\text { ajustadas en }>65 a\end{array}$ & $\begin{array}{c}14 \\
351\end{array}$ & 9747 & $\begin{array}{c}20 \\
163\end{array}$ & 72404,7 & 49176,0 & 101726,0 \\
\hline $\begin{array}{l}\text { Muertes por ERA según } \\
\text { Bogotá en>de } 65 a\end{array}$ & 285 & 200 & 427 & & & \\
\hline $\begin{array}{l}\text { Muertes por ERA según } \\
\text { Manizales en>de } 65 a\end{array}$ & 436 & 306 & 501 & & & \\
\hline Totales & & & & 73377,0 & 49538,4 & 104194,8 \\
\hline
\end{tabular}


Tabla 2. Análisis de sensibilidad usando estimados de Bogota o Manizales Colombia 2007

\begin{tabular}{|c|c|c|c|c|c|c|}
\hline \multirow{2}{*}{ Parámetros } & \multicolumn{3}{|c|}{ Bogotá } & \multicolumn{3}{|c|}{ Manizales } \\
\hline & Base & $\operatorname{Lim} \operatorname{Inf}$ & Lim sup & Base & $\operatorname{Lim} \operatorname{Inf}$ & Lim sup \\
\hline \multicolumn{7}{|c|}{ Con parámetros mínimos de efectividad de la vacuna y a los costos mas altos de vacunación } \\
\hline $\begin{array}{l}\text { Muertes evitadas en }<\text { de } \\
\text { dos años }\end{array}$ & 87 & 79 & 162 & 47 & 37 & 157 \\
\hline $\begin{array}{l}\text { Muertes evitadas en }>\text { de } 65 \\
\text { años }\end{array}$ & 271 & 190 & 407 & 425 & 298 & 488 \\
\hline $\begin{array}{l}\text { AVAD evitados en }<\text { de dos } \\
\text { años }\end{array}$ & 6.090 & 5.516 & 11.326 & 3.318 & 2.562 & 10.990 \\
\hline $\begin{array}{l}\text { AVAD evitados en > de } 65 \\
\text { años }\end{array}$ & 1.356 & 950 & 2.034 & 2.126 & 1.492 & 2.442 \\
\hline $\begin{array}{l}\text { ICER/ AVAD evitado } \\
\text { (U\$/AVAD) en < de } 2 a\end{array}$ & 661 & 221 & 842 & 1.212 & 227 & 1.813 \\
\hline $\begin{array}{l}\text { ICER/ muerte evitada } \\
\text { (U\$/muerte) en }<\text { de } 2 a\end{array}$ & 2.967 & 1.228 & 4.890 & 1.892 & 1.023 & 3.113 \\
\hline $\begin{array}{l}\text { ICER/ AVAD evitado } \\
\text { (U\$/AVAD) en > de } 65 a\end{array}$ & -43.233 & -43.735 & -36.415 & -27.575 & -36.428 & -23.187 \\
\hline $\begin{array}{l}\text { ICER/ muerte evitada } \\
\text { (U } \$ / \text { muerte) en }>\text { de } 65 a\end{array}$ & -216.166 & -218.674 & -182.077 & -137.875 & -182.139 & -115.934 \\
\hline $\begin{array}{l}\text { ICER/ AVAD evitado } \\
\text { (U\$/AVAD) totales }\end{array}$ & -7.333 & -6.471 & -4.632 & -10.030 & -6.437 & -7.388 \\
\hline $\begin{array}{l}\text { ICER/ muerte evitada } \\
\text { (U\$/muerte) totales }\end{array}$ & -152.433 & -152.055 & -111.419 & -115.534 & -133.961 & -89.402 \\
\hline \multicolumn{7}{|c|}{ Con parámetros máximos de efectividad de la vacuna y a los costos mas bajos de vacunación } \\
\hline $\begin{array}{l}\text { Muertes evitadas en }<\text { de } \\
\text { dos años }\end{array}$ & 104 & 95 & 194 & 57 & 44 & 188 \\
\hline $\begin{array}{l}\text { Muertes evitadas en }>\text { de } 65 \\
\text { años }\end{array}$ & 292 & 204 & 437 & 457 & 321 & 525 \\
\hline $\begin{array}{l}\text { AVAD evitados en }<\text { de dos } \\
\text { años }\end{array}$ & 7.308 & 6.619 & 13.591 & 3.982 & 3.074 & 13.188 \\
\hline $\begin{array}{l}\text { AVAD evitados en }>\text { de } 65 \\
\text { años }\end{array}$ & 1.458 & 1.021 & 2.187 & 2.285 & 1.604 & 2.625 \\
\hline $\begin{array}{l}\text { ICER/ AVAD evitado } \\
\text { (U\$/AVAD) en < de } 2 a\end{array}$ & 328 & 68 & 453 & 603 & 71 & 975 \\
\hline $\begin{array}{l}\text { ICER/ muerte evitada } \\
\text { (U } \$ / \text { muerte) en < de } 2 a\end{array}$ & 1.646 & 426 & 2.934 & 1.050 & 354 & 1.868 \\
\hline $\begin{array}{l}\text { ICER/ AVAD evitado } \\
\text { (U\$/AVAD) en > de } 65 a\end{array}$ & -40.129 & -39.931 & -34.926 & -25.595 & -33.260 & -22.238 \\
\hline $\begin{array}{l}\text { ICER/ muerte evitada } \\
\text { (U\$/muerte) en }>\text { de } 65 a\end{array}$ & -200.646 & -199.657 & -174.630 & -127.976 & -166.299 & -111.192 \\
\hline $\begin{array}{l}\text { ICER/AVAD evitado } \\
\text { (U\$/AVAD) totales }\end{array}$ & -6.400 & -5.475 & -4.276 & -8.951 & -5.463 & -6.984 \\
\hline $\begin{array}{l}\text { ICER/ muerte evitada } \\
\text { (U\$/muerte) totales }\end{array}$ & -141.681 & -136.794 & -109.338 & -109.145 & -121.079 & -89.584 \\
\hline
\end{tabular}

La Tabla 3 muestra que la circulación de influenza en Colombia puede ser extremadamente irregular con años en que la circulación del virus persiste por 4 a 6 meses y otros en que la duración de la circulación del virus es mucho más corta. Es de esperarse que los costos y las consecuencias epidemiológicas de la circulación del virus sean mayores en los años de mayor circulación mientras que se reducirían ostensiblemente en los periodos de temporadas cortas. Este 
comportamiento del virus también apoya la idea de que los estimados del presente estudio representarían un promedio conservador del impacto del virus de influenza en Colombia.

Un estudio de Fuchs S, et al., (13), muestra que la carga de muerte por ERA por influenza en niños menores de 5 años en países como Perú, Guyana y Bolivia, es equivalente a $15 \%$ al $20 \%$ del total de muertes. Halpern R, et al encontraron que en Brasil (14), del $25 \%$ al $33 \%$ del número total de muertes observadas en los primeros cinco años de vida son debidas a Influenza. Nuestro estudio estimó que el total de muertes atribuibles a influenza equivale al 30,2 \% del total de muertes por enfermedad respiratoria en niños menores de 2 años, lo que coincide con los estimados mencionados arriba y con los de de Fleming et al en Estados Unidos (15) y los de Kuri-Morales (10) en México.

Tabla 3. Temporadas de circulación $(X)$ del Virus de Influenza en Colombia, 1997 a 2005

\begin{tabular}{|c|c|c|c|c|c|c|c|c|c|c|c|c|c|}
\hline Años & Ene & Feb & $\begin{array}{c}\mathrm{Ma} \\
\mathrm{r}\end{array}$ & Abr & May & Jun & Jul & Ago & Sep & Oct & $\begin{array}{c}\text { No } \\
v\end{array}$ & Dic & Serotipo predominante \\
\hline 1997 & & & & & & & & & $x$ & $x$ & & & Influenza A H3N2 \\
\hline 1998 & & & & & & & & & $x$ & $\mathrm{x}$ & $x$ & & Influenza A H3N2 \\
\hline 1999 & & & & & $x$ & $x$ & $x$ & $\mathrm{x}$ & $x$ & $\mathrm{X}$ & $x$ & $\mathrm{x}$ & $\begin{array}{c}\text { Influenza A H3N2 + } \\
\text { Influenza B }\end{array}$ \\
\hline 2000 & & & & $x$ & $x$ & $x$ & & & & & & & Influenza H1N1 \\
\hline 2001 & & & & & & & & & & & & & No circuló \\
\hline 2002 & & & & & & & $x$ & $\mathrm{x}$ & $x$ & $x$ & & & $\begin{array}{c}\text { Influenza A H3N2 + } \\
\text { Influenza B }\end{array}$ \\
\hline 2003 & & & & & & & & & & $x$ & $x$ & $x$ & Influenza A H3N2 \\
\hline 2004 & & & & & $x$ & $x$ & $\mathrm{x}$ & $x$ & $x$ & $\mathrm{x}$ & $x$ & & $\begin{array}{c}\text { Influenza A H3N2 + } \\
\text { Influenza B }\end{array}$ \\
\hline 2005 & & & & & & & & & $x$ & $x$ & $x$ & & Influenza B \\
\hline
\end{tabular}

De acuerdo a nuestros datos se esperan en los niños menores de 2 años, 4 310 casos de ERA atribuibles a influenza, los cuales se encuentran dentro del intervalo de confianza calculado con los datos del SIS 12 que fueron utilizados como validadores (6 959 casos IC $95 \% 2345$ - 7 896). Esto indica que nuestros cálculos de casos aunque son conservadores son muy parecidos a los datos realmente observados en el período 1990 a 1997. Para los mayores de 65 años utilizando los datos de diferentes estudios $(12,17)$, se calculan 8654 casos de ERA atribuibles a influenza y con la vacunación podrían evitarse entre 3462 y 4 327 casos en este grupo de edad.

Los resultados del análisis de costos efectividad de la vacunación en menores de dos años, muestra una razón alta de costo efectividad de la vacuna frente a los parámetros de OMS lo cual es consistente con lo esperado para este grupo y 
menor que para los grupos de alto riesgo en donde la vacuna ha mostrado ser costo ahorrativa (18). Respecto a los mayores de 65 años la vacunación resultó ser costo ahorrativo con resultados similares a otros estudios (19-22). En el análisis de sensibilidad los resultados continúan siendo costo efectivos para menores de dos años y costos ahorrativos para mayores de 65 en cualquiera de los dos escenarios usados, pese a que hay una importante variación entre ellos, lo que sugiere que los estimados presentados aquí son robustos frente a variaciones de los estimadores epidemiológicos.

Varios estudios muestran que la vacunación de menores de dos años reduce la morbilidad y mortalidad asociada a influenza en adultos mayores (16,19-21). La inclusión de este efecto rebaño puede incrementar dramáticamente el costo efectividad de la vacunación en niños (20). El presente estudio no incluye el potencial efecto rebaño pero los resultados, sin este efecto, son suficientemente buenos como para justificar la inclusión de la vacuna en los mayores de 65 años.

En conclusión, los resultados de nuestro estudio muestran que la carga de enfermedad por influenza en Colombia es muy importante en dos de las poblaciones de mayor riesgo y que la introducción de la vacuna esta justificada. Una recomendación adicional tiene que ver con la necesidad de reforzar el esquema de vigilancia sobre la circulación del virus ampliando el número de ciudades que participan en la identificación de los virus circulantes. Dado que Colombia ya ha introducido la vacunación universal en menores de dos años, también se hace necesario el iniciar estudios de evaluación de la efectividad de esta política ya que la evidencia sobre el impacto de la vacuna inactivada en niños es escasa en todo el mundo

Agradecimientos. Agradecemos al Laboratorio de Virologia del Instituto Nacional de Salud y su coordinadora, Dra. Gloria Rey, el acceso que nos brindo a los resultados de la vigilancia sobre virus respiratorios y la colaboración que brindo en la interpretación de esos resultados.

\section{REFERENCIAS}

1. Molinari NA, Ortega-Sanchez IR, Messonnier ML, Thompson WW, Wortley PM, Weintraub E, et al. The annual impact of seasonal influenza in the US: measuring disease burden and costs. Vaccine. 2007 Jun 28:25(27):5086-96.

2. Keech $M$, Beardsworth $P$. The impact of influenza on working days lost: a review of the literature. Pharmacoeconomics. 2008;26(11):911-24.

3. WHO. H5N1 avian influenza: first steps towards development of a human vaccine [Internet]. Geneve. 2005. Disponible en:http://www.who.int/csr/disease/avian_influenza/statement_2005_08_12/en/index.html Consultado: mayo 2009. 
4. Ministerio de la Protección Social, República de Colombia. La influenza y su situación en Colombia. Dirección General de Salud Pública, Grupo de Vigilancia en Salud Pública. Bogota DC; 2005.

5. Abarca K. Influenza: Vacunación a nuevos grupos etarios. Rev chil infectol. 2007:24(3):227-30.

6. Bridges CB, Harper SA, Fukuda K, Uyeki TM, Cox NJ, Singleton JA. Prevention and control of influenza. Recommendations of the Advisory Committee on Immunization Practices (ACIP). MMWR Recomm Rep. 2003 Apr 25;52(RR-8):1-34; quiz CE1-4.

7. Monto A S. Global burden of influenza: what we know and what we need to know. International Congress Series 1263 2004;3-11.

8. Shek LP, Lee BW. Epidemiology and seasonality of respiratory tract virus infections in the tropics. Pediatr Respir Rev. 2003 Jun;4(2):105-11.

9. Porras A, Rico A, De la Hoz F, Moreno J, Cotes K,. Mortalidad asociada a la circulación del virus de influenza estacional en Bogotá (Colombia), 1997-2005. Revista Panamericana de Salud Pública. En prensa. 2008; 25 (11).

10. Borrero I, Fajardo L, Bedoya A, Zea A, Carmona F, de Borrero MF. Acute respiratory tract infections among a birth cohort of children from Cali, Colombia, who were studied through 17 months of age. Rev Infect Dis. 1990 Nov-Dec;12 Suppl 8:S950-6.

11. PROFAMILIA. Encuesta Nacional de Demografía y Salud Bogota D.C; 2005.

12. Moreno J, De la Hoz F, Rico A, Cotes K, Porras A. Efectividad de la vacuna contra influenza: metanálisis de literatura. Biomédica. 2009;9(1):87-97.

13. Fuchs SC, Fischer GB, Black RE, Lanata C. The burden of pneumonia in children in Latin America. Paediatr Respir Rev. 2005 Jun;6(2):83-7.

14. Halpern R, Halpern S, Glugliani ERJ. Doença respiratória aguda na criança. In: Duncan BB, Schimidt MI, Glugliani ERJ, editors. Medicina ambulatorial: condutas clínicas em atenção primária. Porto Alegre: Artes Médicas; 1990. p. 336-40.

15. Fleming DM, Pannell RS, Elliot AJ, Cross KW. Respiratory illness associated with influenza and respiratory syncytial virus infection. Arch Dis Child. 2005 Jul;90(7):741-6.

16. Kuri-Morales P, Galván F, Cravioto P, Rosas LAZ, Tapia-Conyer R. Mortalidad en México por influenza y neumonía (1990-2005). Salud Pública de México. 2006;48:379-84.

17. Chiu SS, Lau YL, Chan KH, Wong WH, Peiris JS. Influenza-related hospitalizations among children in Hong Kong. N Engl J Med. 2002 Dec 26;347(26):2097-103.

18. WHO. Report of the Commission on the macroeconomics and health: Investing in Health for economic developments. Geneva: World Health Organization; 2001 Contract No.: Document Number.

19. Dayan GH, Nguyen VH, Debbag R, Gomez R, Wood SC. Cost-effectiveness of influenza vaccination in high-risk children in Argentina. Vaccine. $2001 \mathrm{Jul}$ 20;19(30):4204-13.

20. Nichol KL, Goodman M. Cost effectiveness of influenza vaccination for healthy persons between ages 65 and 74 years. Vaccine. 2002 May 15;20 Suppl 2:S21-4.

21. Facanha M. Impacto da vacinacao de maiores de 60 anos para influenza sobre as internacoes e óbitos por doencas respiratórias e circulatórias em Fortaleza, CE, Brasil. J Bras Pneumo,. 2005;31(5):407-12.

22. Alvis N, De la Hoz F, Higuera AB, Pastor D, Di Fabio JL. The economic costs of pneumonia in children under 2 years of age in Colombia. Rev Panam Salud Publica. 2005 Mar;17(3):178-83.

23. WHO. Informe sobre la salud en el mundo 2002-Reducir los riesgos y promover una vida sana.. Organización Mundial de la Salud; 2002.

24. Dirección General de Salud Pública. Guía técnica para la campaña de vacunación antigripal 2004. Generalidad de Cataluña, Departamento de Sanidad y Seguridad Social; 2004. 